\title{
Clinical significance of inflammatory cytokine and chemokine expression in hand, foot and mouth disease
}

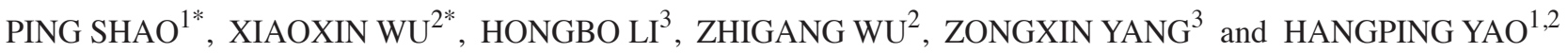 \\ ${ }^{1}$ Department of Infectious Diseases; ${ }^{2}$ State Key Laboratory for Diagnosis and Treatment of Infectious Diseases, \\ Collaborative Innovation Center for Diagnosis and Treatment of Infectious Diseases, First Affiliated Hospital, \\ Zhejiang University School of Medicine, Hangzhou, Zhejiang 310003; ${ }^{3}$ Department of Infectious Diseases, \\ Hangzhou Sixth People's Hospital, Hangzhou, Zhejiang 310014, P.R. China
}

Received December 22, 2015; Accepted January 19, 2017

DOI: $10.3892 / \mathrm{mmr} .2017 .6324$

\begin{abstract}
The present study examined the relationship between cytokine and chemokine expression and the clinical presentation of hand, foot and mouth disease (HFMD), which is currently unclear. The present study involved 28 patients with mild HFMD, 44 patients with severe HFMD and 26 healthy children. Venous blood was tested for cytokine [interleukin (IL)-4, IL-12, IL-18, tumor necrosis factor- $\alpha$ $(\mathrm{TNF}-\alpha)$, interferon- $\gamma($ IFN- $\gamma)]$ and chemokine expression [IL-8, regulated on activation, normal $\mathrm{T}$ cell expressed and secreted (RANTES), monocyte chemoattractant protein-1 (MCP-1) and IFN- $\gamma$-inducible protein-10 (IP-10)]. Stool samples from the patients were tested for enterovirus 71 (EV71) RNA using reverse transcription-polymerase chain reaction. The results indicated that all cytokine/chemokine levels were increased in patients with severe HFMD compared with in patients with mild HFMD or control subjects. In addition, RANTES, MCP-1, IL-4, IL-12 and IL-18 levels were higher in mild HFMD patients than in the controls. In patients with severe HFMD, all expression levels (with the exception of IL-8 and IL-4) were increased in patients with encephalitis plus pulmonary edema compared with those with encephalitis alone. Furthermore, all levels (with the exception of IL-8) were increased in EV71-positive patients compared with EV71-negative patients. In mild HFMD, all levels (with the exception of IL-8 and IL-4) were increased in EV71-positive patients compared with EV71-negative patients.
\end{abstract}

Correspondence to: Dr Hangping Yao, State Key Laboratory for Diagnosis and Treatment of Infectious Diseases, Collaborative Innovation Center for Diagnosis and Treatment of Infectious Diseases, First Affiliated Hospital, Zhejiang University School of Medicine, 79 Qingchun Road, Hangzhou, Zhejiang 310003, P.R. China

E-mail: yaohangping@zju.edu.cn

*Contributed equally

Key words: hand, foot and mouth disease, enterovirus inflammatory cytokines, chemokines, clinical
However, in severe HFMD, only RANTES, IP-10 and IFN- $\gamma$ levels were increased in EV71-positive patients compared with EV71-negative patients. In the EV71-negative group, all levels were increased in severe HFMD compared with mild HFMD. In the EV71-positive group, all levels (with the exception of IL-8) were increased in severe HFMD compared with mild HFMD. These results indicated that cytokines and chemokines participate in HFMD pathogenesis, and may be useful to monitor disease progression and predict prognosis.

\section{Introduction}

Hand, foot and mouth disease (HFMD) is a common acute infectious disease caused by various human intestinal viruses. HFMD usually affects preschool children (age, $<3$ years) and has caused widespread epidemics in this population (1). It is commonly caused by enterovirus 71 (EV71) and coxsackievirus A16 (1) and EV71 leads to more HFMD compared with coxsackievirus A16. Meanwhile, EV71 is the most neurovirulent of enteroviruses and is responsible for fatal outcomes. EV71 is a member of an enterovirus group that belongs to the Picornaviridae family. Similar to other members of Picornaviridae, it is a non-enveloped, positive, non-segmented RNA virus, with a diameter of $\sim 30 \mathrm{~nm}$. Its genomic RNA is packaged within the capsid composed of 60 copies of 4 structural proteins, VP1, VP2, VP3 and VP4. It was first isolated in California, in 1969. Since the 1960s, EV71 has led to numerous epidemic outbreaks worldwide. Presently, it has become a major public health issue, especially in Asia-Pacific region (2).

The severity of HFMD varies; patients with mild HFMD present with mild fever and a self-limiting herpetic rash on the hands, feet and mouth. However, in patients with severe HFMD, the disease progresses rapidly and may involve meningitis, brainstem encephalitis, pulmonary angioneurotic edema, pulmonary hemorrhage, myocarditis, and even cardiopulmonary failure and mortality $(3,4)$. Good oral and skin hygiene is crucial for patients with mild HFMD. Additionally, quaran71, tine of infected individuals is also effective in order to limit the spread of the virus. Symptomatic supportive treatments should be conducted when required. No established antiviral treatments are available for severe HFMD. Intravenous 
immunoglobulin treatment has been reported to be effective and is used in various Asian countries; however, sufficient evidence for its effectiveness is lacking. Glucocorticoid may also be used to reduce brain and pulmonary edema. A comprehensive life support including fluid management, inotrope and respiratory support therapy is regularly conducted (5-7).

The pathogenesis of severe HFMD caused by EV71 infection is not fully understood. Numerous studies have demonstrated that cellular and humoral immune dysfunction in patients with HFMD, in particular the abnormal expression of inflammatory cytokines and chemokines and an imbalance in the expression of anti- and proinflammatory cytokines, is involved in the exacerbation of HFMD (8-11).

In the present study, the expression levels of inflammatory cytokines and chemokines were detected in peripheral blood samples obtained from children with EV71-induced HFMD. The relationship between abnormal cytokine/chemokine expression and various clinical presentations of HFMD was analyzed in order to clarify the mechanisms underlying the exacerbation of HFMD. The clinical significance of the results is discussed.

\section{Materials and methods}

Patient selection. The present study involved 72 children (39 male, 33 female) with mild or severe HFMD who were treated at Hangzhou Sixth People's Hospital (Hangzhou, China) between March 2011 and June 2013. All diagnoses were made using the Foot and Mouth Disease Treatment Guidelines (2010) issued by the Ministry of Health and the Expert Consensus on the Clinical Treatment of Severe EV71 Infection (6,7). Patients who received immunomodulators, or who had other viral or bacterial infections or other immune disorders, were excluded.

In addition, 26 healthy children (control group), who had normal findings on physical examination from Hangzhou Sixth People's Hospital, were recruited. Verbal consent was obtained from all participants. Ethical approval (no. 2017-11) for the present study was obtained from the Ethics Committee of the First Affiliated Hospital, College of Medicine, Zhejiang University (Hangzhou, China).

Sample collection. Venous blood samples $(3 \mathrm{ml})$ were collected from all children in the HFMD and control groups within $24 \mathrm{~h}$ of admission. The blood samples were collected in EDTA tubes and centrifuged at 3,000 $\mathrm{x} g$ for $15 \mathrm{~min}$ at room temperature to obtain plasma samples, which were subsequently stored at $-80^{\circ} \mathrm{C}$ until further analysis. In addition, stool samples were collected from the children with HFMD, and these were subjected to reverse transcription-polymerase chain reaction (RT-PCR) analysis for the detection of EV71 RNA.

EV71 detection. A diagnostic PCR-fluorescence probing kit (cat. no. DA-BT109) for enterovirus RNA (Da An Gene Co., Ltd., Guangzhou, China) was used for the detection of EV71. The RNA extraction reagent was conducted in the PCR-fluorescence probing kit. All forward and reverse primer sequences used were contained within the kit. The procedure was conducted according to the manufacturer's protocol. The thermal cycling parameters were as follows: cDNA synthesis at $42^{\circ} \mathrm{C}$ for $25 \mathrm{~min}$; denaturation at $94^{\circ} \mathrm{C}$ for $3 \mathrm{~min}$; and 40 cycles at $93^{\circ} \mathrm{C}$ for $15 \mathrm{sec}$ and $55^{\circ} \mathrm{C}$ for $45 \mathrm{sec}$. RT-PCR was performed on the CFX96 ${ }^{\mathrm{TM}}$ Real-Time PCR Detection system (Bio-Rad Laboratories, Inc., Hercules, CA, USA). If test sample had a typical S-shaped amplification curve and the $\mathrm{Cq}$ value (12) was lower than 34.9 , it was deemed to be positive, otherwise it was negative.

Cytokine and chemokine detection. Plasma expression levels of the cytokines interleukin (IL)-4, IL-12, IL-18, tumor necrosis factor- $\alpha$ (TNF- $\alpha)$ and interferon- $\gamma$ (IFN- $\gamma$ ), and the chemokines IL-8, regulated on activation, normal $\mathrm{T}$ cell expressed and secreted (RANTES), monocyte chemoattractant protein-1 (MCP-1) and IFN- $\gamma$-inducible protein-10 (IP-10) were determined using double-antibody sandwich enzyme-linked immunosorbent assays (ELISA; R\&D Systems, Inc., Minneapolis, MN, USA), according to the manufacturer's protocols. The catalogue numbers for all ELISA kits used were as follows: IL-4, D4050; IL-12, D1200; IL-18, 7620; TNF- $\alpha$, DTA00C; IFN- $\gamma$, DIF50; IL-8, D8000C; RANTES, DRN00B; MCP-1, DCP00; IP-10, DIP100.

Statistical analysis. Data were analyzed using SPSS 13.0 statistical software (SPSS, Inc., Chicago, IL, USA) and were representative of three independent experiments expressed as the mean \pm standard deviation. Multi-sample means were detected using one-way ANOVA analysis of variance and the Student's t-test. The multiple comparisons test was adjusted by Bonferroni test. Correlations were analyzed using Pearson's correlation analysis. $\mathrm{P}<0.05$ was considered to indicate a statistically significant difference.

\section{Results}

Demographic data. Of the 72 study participants (39 male, 33 female), 28 (17 male, 11 female; average age, $2.3 \pm 0.8$ years) had mild HFMD and 44 (22 male, 22 female; average age, $2.1 \pm 0.7$ years) had severe HFMD. The control group included 16 males and 10 females, with an average age of $2 \pm 0.6$ years. No significant differences in age or gender were present between the mild HFMD, severe HFMD and control groups.

Cytokine and chemokine expression. The plasma expression levels of all chemokines (IL-8, RANTES, MCP-1, IP-10; Fig. 1A) and cytokines (IL-4, IL-12, IL-18, TNF- $\alpha$, IFN- $\gamma$; Fig. 1B) were significantly increased in the severe HFMD group compared with the mild HFMD and normal control groups $(\mathrm{P}<0.01)$. In addition, the expression levels of the chemokines RANTES and MCP-1 (Fig. 1A) and the cytokines IL-4, IL-12 and IL-18 (Fig. 1B) were significantly increased in the mild HFMD group compared with the normal control group $(\mathrm{P}<0.05)$.

The children in the severe HFMD group were further divided into those with encephalitis (E group; $n=26 ; 14$ male, 12 female) and those with both encephalitis and pulmonary edema ( $\mathrm{E}+\mathrm{P}$ group; $\mathrm{n}=18 ; 8$ male, 10 female). No significant differences in age or gender were present between the $\mathrm{E}$ and $\mathrm{E}+\mathrm{P}$ groups. The plasma expression levels of all chemokines, with the exception of IL-8 (Fig. 2A), and all cytokines, with 
A

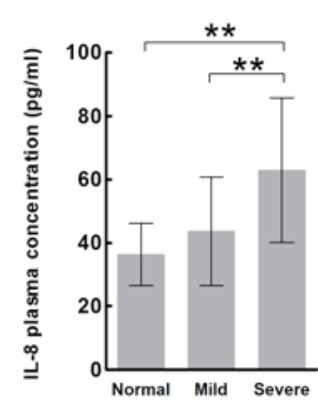

B
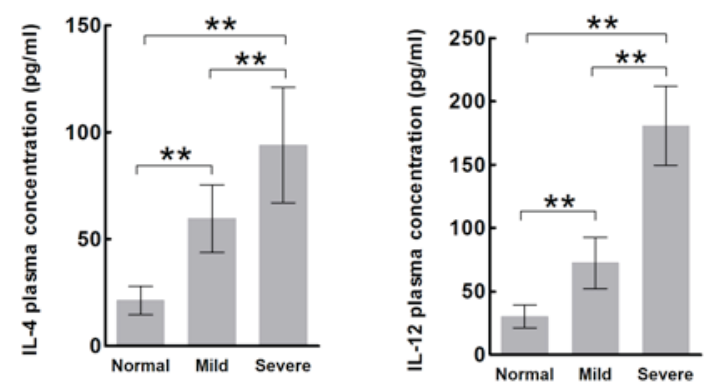
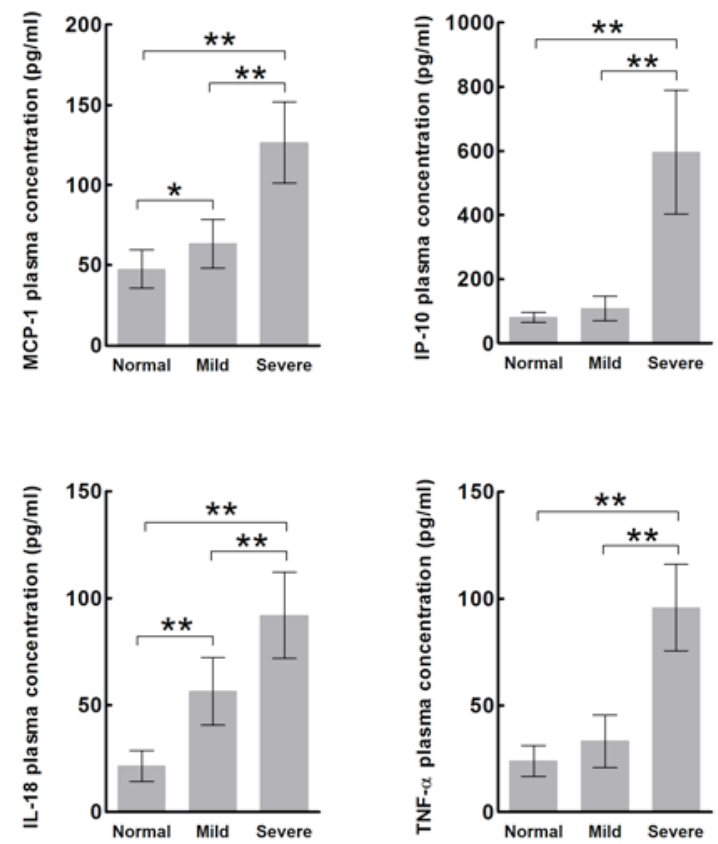

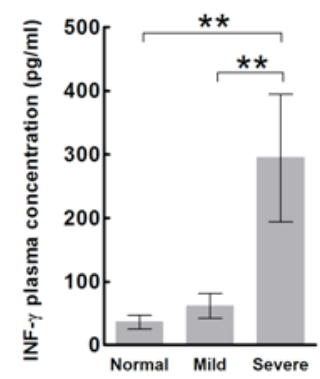

Figure 1. Expression of inflammatory (A) chemokines and (B) cytokines in children with severe and mild hand, foot and mouth disease. ${ }^{*} \mathrm{P}<0.05$, ${ }^{* *} \mathrm{P}<0.01$. IL, interleukin; RANTES, regulated on activation, normal T cell expressed and secreted; MCP-1, monocyte chemoattractant protein-1; IP-10, IFN- $\gamma$-inducible protein-10; TNF- $\alpha$, tumor necrosis factor- $\alpha$; IFN- $\gamma$, interferon- $\gamma$.

the exception of IL-4 (Fig. 2B) were significantly increased in the $\mathrm{E}+\mathrm{P}$ group compared with the $\mathrm{E}$ group $(\mathrm{P}<0.05)$.

EV71 RNA detection in stool samples and its correlation with plasma cytokine and chemokine expression. Stool samples from the children with HFMD were analyzed for the presence of EV71 RNA using RT-PCR (Table I). The plasma expression levels of all chemokines, with the exception of IL-8 (Fig. 3A), and all cytokines (Fig. 3B) were significantly increased in children whose stool samples tested positive for EV71 RNA compared with children whose stool samples were negative for EV71 RNA $(\mathrm{P}<0.05)$. In the mild HFMD group, the plasma expression levels of all chemokines, with the exception of IL-8 (Fig. 4A), and all cytokines, with the exception of IL-4 (Fig. 4B) were significantly increased in the EV71-positive patients compared with the EV71-negative patients $(\mathrm{P}<0.05)$. However, in the severe HFMD group, only the expression levels of RANTES (Fig. 4A), IP-10 and IFN- $\gamma$ (Fig. 4B) were significantly increased in the EV71-positive patients compared with the EV71-negative patients $(\mathrm{P}<0.05)$.

The children with HFMD were regrouped according to their EV71 status. Of the 72 study patients, 33 (18 male, 15 female) were EV71 negative, and 39 (21 male, 18 female) were EV71 positive. Of the 33 EV71-negative patients, 17 (10 male, 7 female) had mild HFMD and 16 ( 8 male, 8 female) had severe HFMD. Of the 39 EV71-positive patients, 11 ( 7 male, 4 female) had mild HFMD and 28 (14 male, 14 female) had severe HFMD (Table I).

In the EV71-negative group, the plasma expression levels of all cytokines (Fig. 5A) and chemokines (Fig. 5B) were significantly increased in children with severe HFMD compared with children with mild HFMD $(\mathrm{P}<0.01)$. In the EV71-positive group, the plasma expression levels of all cytokines, with the exception of IL-8 (Fig. 5A), and all chemokines (Fig. 5B) were significantly increased in children with severe HFMD compared with children with mild HFMD $(\mathrm{P}<0.01)$.

\section{Discussion}

The immune system is a complex, sophisticated and coordinated network, and its dynamic equilibrium sustains health. Upon invasion by microorganisms, inflammatory mediators are generated in 'cascades' through a series of pathways, which results in systemic inflammatory response syndrome. In addition, the excessive generation of inflammatory mediators may lead to tissue and organ damage; therefore, various 


\section{A}

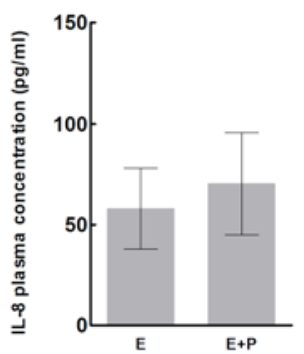

B
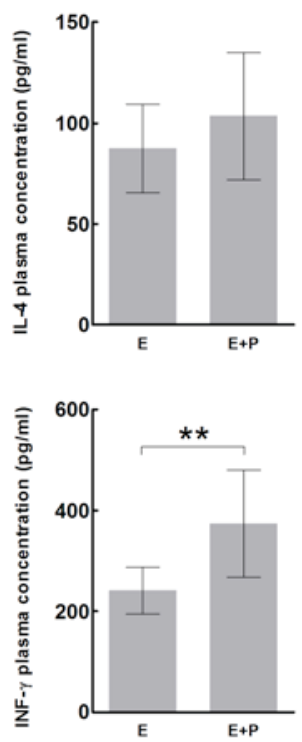
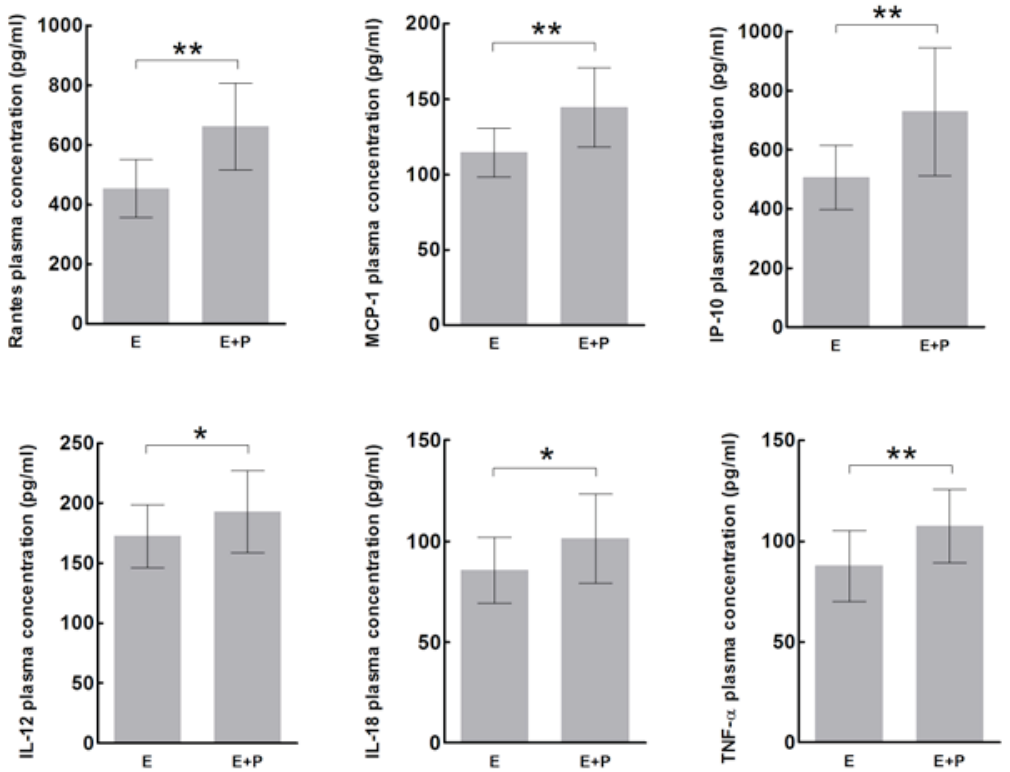

Figure 2. Expression of inflammatory (A) chemokines and (B) cytokines in children with severe hand, foot and mouth disease, in the E and E+P groups. ${ }^{*} \mathrm{P}<0.05,{ }^{* *} \mathrm{P}<0.01$. E, encephalitis group; E+P, encephalitis with pulmonary edema group; IL, interleukin; RANTES, regulated on activation, normal T cell expressed and secreted; MCP-1, monocyte chemoattractant protein-1; IP-10, IFN- $\gamma$-inducible protein-10; TNF- $\alpha$, tumor necrosis factor- $\alpha$; IFN- $\gamma$, interferon- $\gamma$.

anti-inflammatory mediators are produced to inhibit excessive inflammatory responses and protect the body from immune damage (13). Pro- and anti-inflammatory mediators coexist in the infection-induced immunological stress response, and the growth, decline and equilibrium of the two determine the evolution, development and prognosis of infectious disease (14).

In children, the immune system is not fully developed, which facilitates the invasion of pathogenic microorganisms. As a result, children, particularly preschoolers, exist in a physiological immunocompromised state, and their specific and non-specific immune responses are poor compared with those of adults. This may partially explain why preschoolers are susceptible to HFMD.

Following EV71 infection, susceptible cells and non-specific immune cells are stimulated to produce cytokines including IFNs, TNF- $\alpha$ and IL-12. These cytokines are involved in the early control of viral replication and infection. The virus further induces the production of chemokines, including IL-8, MCP-1 and IP-10, which contribute to the recruitment of non-specific immune cells, including natural killer cells, monocytes and macrophages, to the infection site. Furthermore, the activation of these cells by cytokines results in the secretion of inflammatory mediators and cytokines, interference with viral replication and the death of virus-infected host cells. These responses expand and enhance anti-infection immunity and accelerate recovery from HFMD, leading to a self-limiting disease $(10,11,15-17)$. However, the pathogenic mechanisms that lead to the exacerbation of EV71 infection and to abnormal cytokine expression remain unknown.

In the present study, the relationship between plasma cytokines (IL-4, IL-12, IL-18, TNF- $\alpha$, IFN- $\gamma$ ) and chemokines (IL-8, RANTES, MCP-1, IP-10), and disease severity (mild vs. severe groups), complications (E vs. E+P groups) and fecal EV71 status (EV71-positive vs. EV71-negative groups) was examined in children with HFMD. The results demonstrated that cytokine and chemokine expression levels were i) significantly increased in the severe HFMD group compared with the mild HFMD and normal control groups; ii) significantly increased in the $\mathrm{E}+\mathrm{P}$ group compared with the $\mathrm{E}$ group; and iii) significantly increased in the EV71-positive group compared with the EV71-negative group, regardless of disease severity. These results suggested that EV71 infection-induced abnormal expression of cytokines and chemokines was involved in the exacerbation of HFMD.

It has previously been demonstrated that cytokines, including IL-1 $\beta$, IL-6, IL-18, TNF- $\alpha$ and IFN- $\gamma$, have inhibitory effects on the replication and infective ability of numerous viruses, and that these compounds are major components of the early non-specific immune response to viral infections (18). Conversely, chemokines, including IL-8, RANTES, MCP-1 and IP-10, are involved in the recruitment and activation 
Table I. Hand, foot and mouth disease groups and EV71 status.

\begin{tabular}{|c|c|c|c|c|}
\hline \multirow[b]{2}{*}{ Group } & \multicolumn{2}{|c|}{ EV71-PCR (+) } & \multicolumn{2}{|c|}{ EV71-PCR (-) } \\
\hline & No. (M/F) & Average age (y) & No. (M/F) & Average age (y) \\
\hline Mild & $11(7 / 4)$ & $2.3 \pm 0.8$ & $17(10 / 7)$ & $2.2 \pm 0.9$ \\
\hline Severe (E) & $17(8 / 9)$ & $1.9 \pm 0.7$ & $9(6 / 3)$ & $2.1 \pm 0.6$ \\
\hline Severe $(E+P)$ & $11(6 / 5)$ & $2.1 \pm 0.8$ & $7(2 / 5)$ & $2.2 \pm 0.6$ \\
\hline
\end{tabular}

EV71, enterovirus 71; PCR, polymerase chain reaction; M, male; F, female; E, encephalitis; P, pulmonary edema.

A
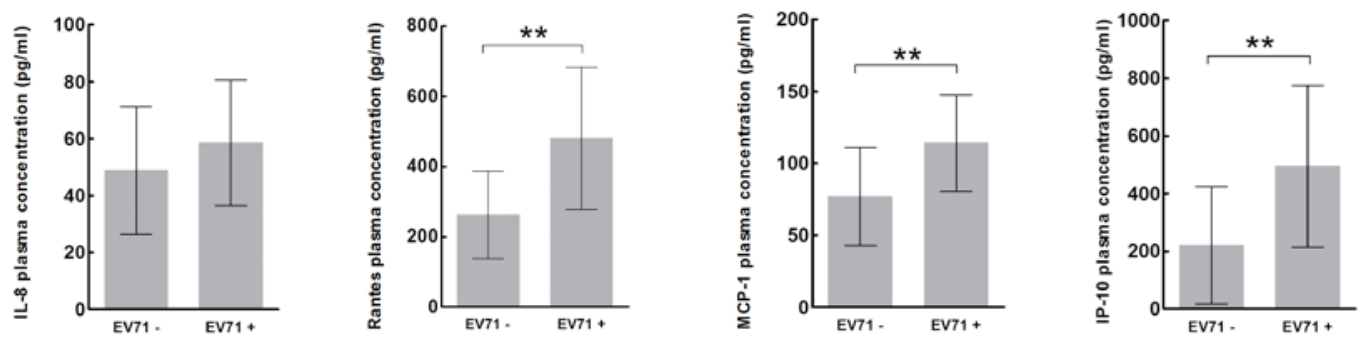

B
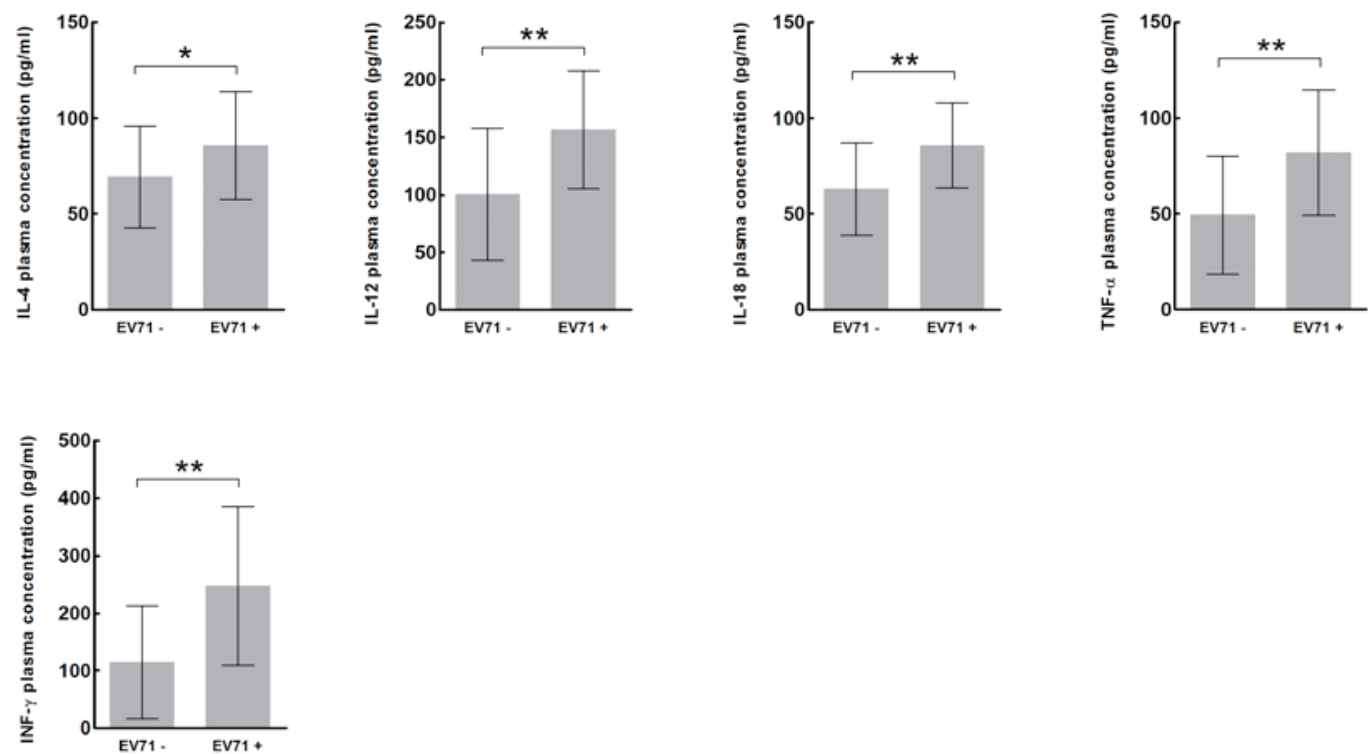

Figure 3. Expression of inflammatory (A) chemokines and (B) cytokines in patients with hand, foot and mouth disease whose stool samples tested positive or negative for EV71 RNA. ${ }^{*} \mathrm{P}<0.05,{ }^{* *} \mathrm{P}<0.01 \mathrm{es}$. EV71, enterovirus 71; IL, interleukin; RANTES, regulated on activation, normal T cell expressed and secreted; MCP-1, monocyte chemoattractant protein-1; IP-10, IFN- $\gamma$-inducible protein-10; TNF- $\alpha$, tumor necrosis factor- $\alpha$; IFN- $\gamma$, interferon- $\gamma$.

of immune cells (19), thereby further enhancing antiviral immunity. Overexpression or imbalances in the expression of these immune factors aggravates the inflammatory response to infection, causing damage to infected tissues and leading to loss of or disordered organ function. Under these circumstances, the vulnerable immune system of children is unable to effectively eliminate the virus and control inflammation, which results in further spread of the virus and eventually causes severe HFMD.

Lin et al (10) confirmed the aforementioned mechanism by demonstrating that the peripheral expression levels of IL-1 $\beta$ and several other inflammatory cytokines were significantly increased in patients with EV71-induced HFMD with both encephalitis and pulmonary edema, as compared with in those with only encephalitis, those with mild symptoms or normal controls. A previous study reported similar results; demonstrating that the expression levels of inflammatory cytokines, including IFN- $\gamma$, MCP-1 and IP-10 in the peripheral blood and cerebrospinal fluid were significantly increased in patients with pulmonary edema compared with patients with encephalitis or patients with mild disease (11). Similar results have been reported for the inflammatory cytokine TNF- $\alpha$, 

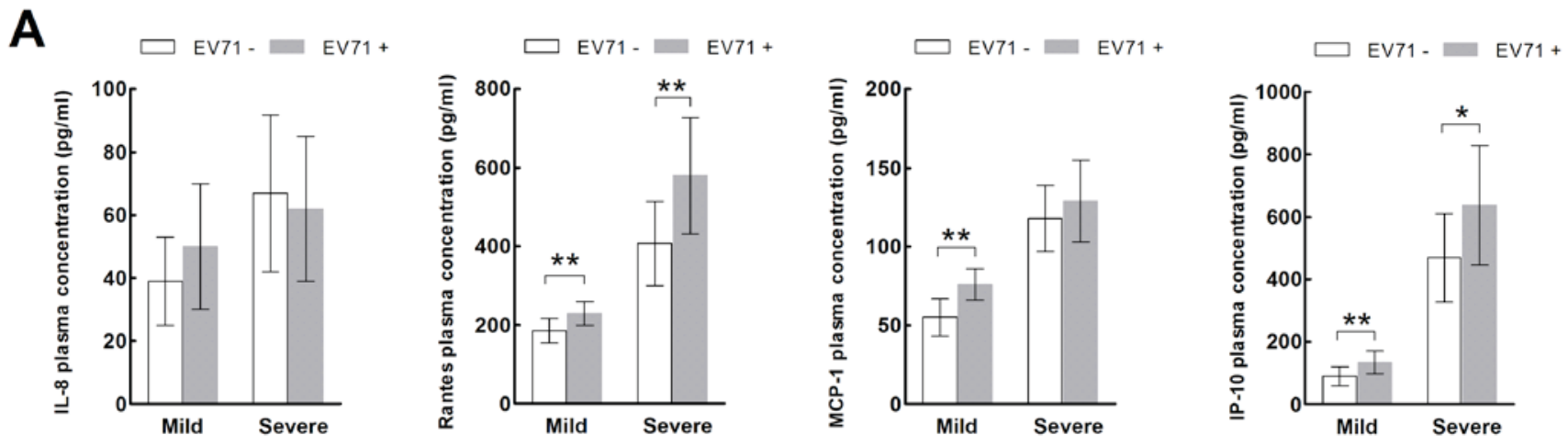

B
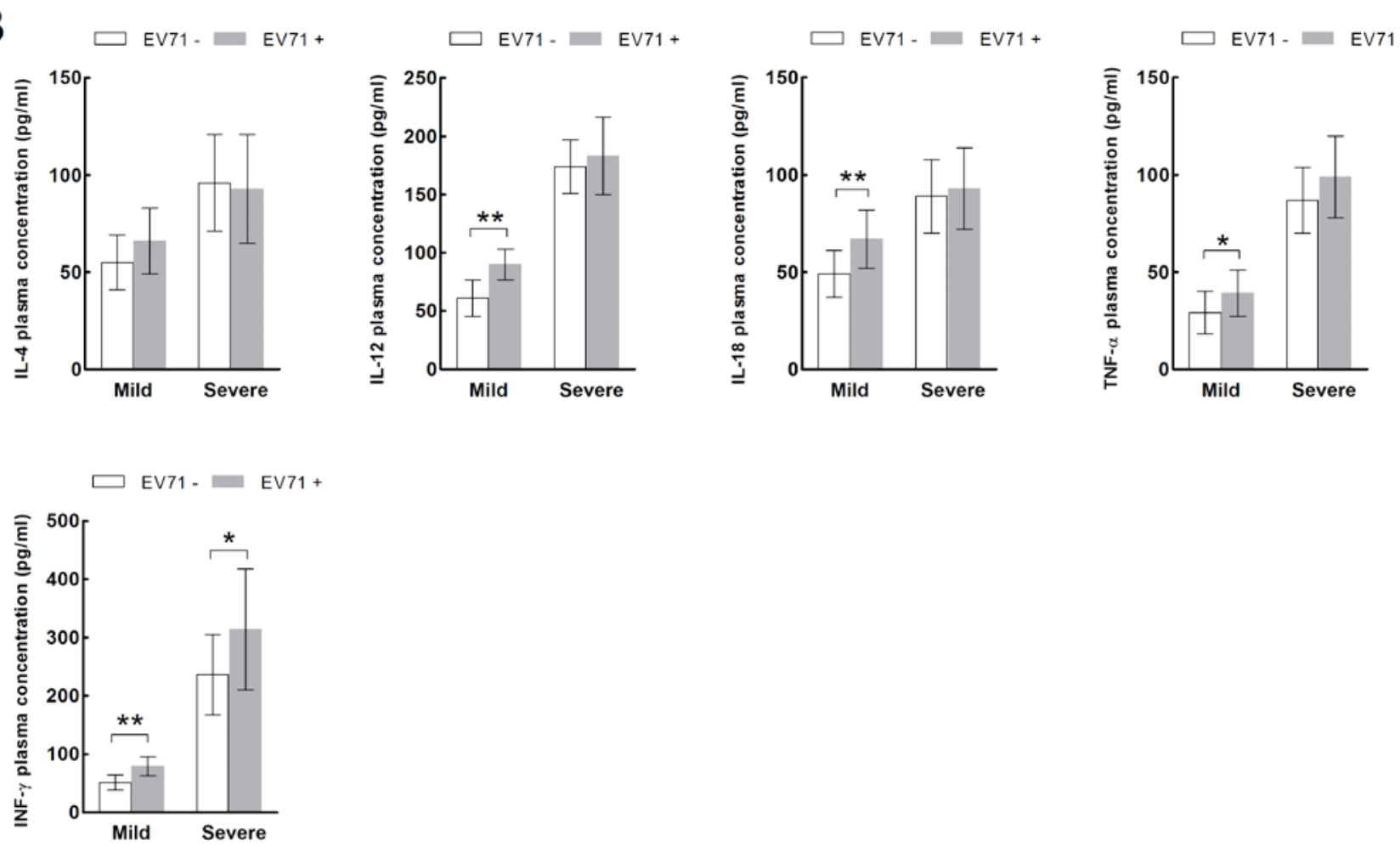

Figure 4. Expression of inflammatory (A) chemokines and (B) cytokines in the mild and severe hand, foot and mouth disease groups according to EV71 status. ${ }^{*} \mathrm{P}<0.05,{ }^{* *} \mathrm{P}<0.01$. EV71, enterovirus 71; IL, interleukin; RANTES, regulated on activation, normal T cell expressed and secreted; MCP-1, monocyte chemoattractant protein-1; IP-10, IFN- $\gamma$-inducible protein-10; TNF- $\alpha$, tumor necrosis factor- $\alpha$; IFN- $\gamma$, interferon- $\gamma$.

which was reported to have an abnormally high expression in patients with HFMD displaying pulmonary edema and encephalitis (20).

These results indicated that in the early stages of infection the increase in the levels of various cytokines and chemokines may contribute to the early activation of specific and non-specific immunity. This maximizes the mobilization of various types of immune cells to participate in anti-EV71 infection immunity for the alleviation of or recovery from the disease. When the immune system fails to effectively clear the virus and control inflammation, the disease is exacerbated. Overexpression of inflammatory cytokines and chemokines in infected tissues induces excessive immune responses and results in cytokine cascade reaction and cytokine storm through autocrine and paracrine mechanisms, resulting in severe local and systemic inflammation, and eventually tissue damage and organ dysfunction.
In conclusion, during the development and progression of HFMD, various cytokines and chemokines are involved in the occurrence of inflammation. Some of these compounds clear the virus, whereas others exacerbate the disease. However, at present, the immune pathogenesis of HFMD, its cytokine expression patterns and the changes in these patterns are not clear. Further investigation into the involvement of HFMD-associated cytokines and chemokines in the pathogenesis of the disease is required to search for cytokine indices to be used to monitor disease progression, determine prognosis, and guide clinical diagnosis and treatment.

\section{Acknowledgements}

The present study was supported in part by grants (grant no. 2014ZZ01) from the State Key Laboratory for Diagnosis and Treatment of Infectious Diseases, China. 
A

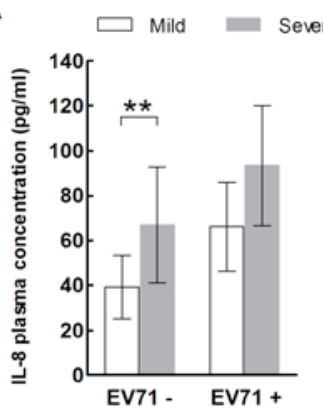

B

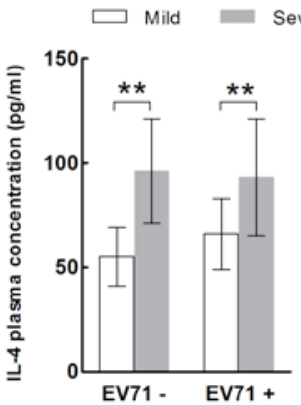

$\square$ Mild $\square$ Severe

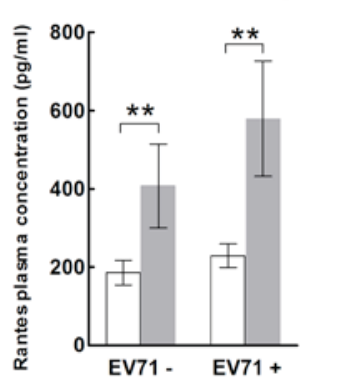

$\square$ Mild $\square$ Severe

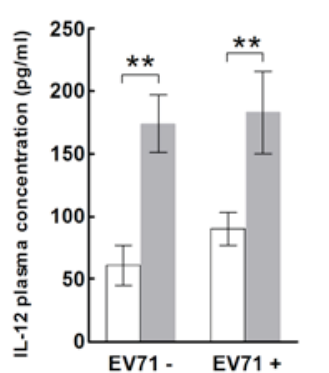

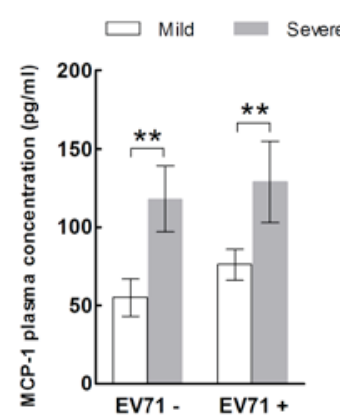
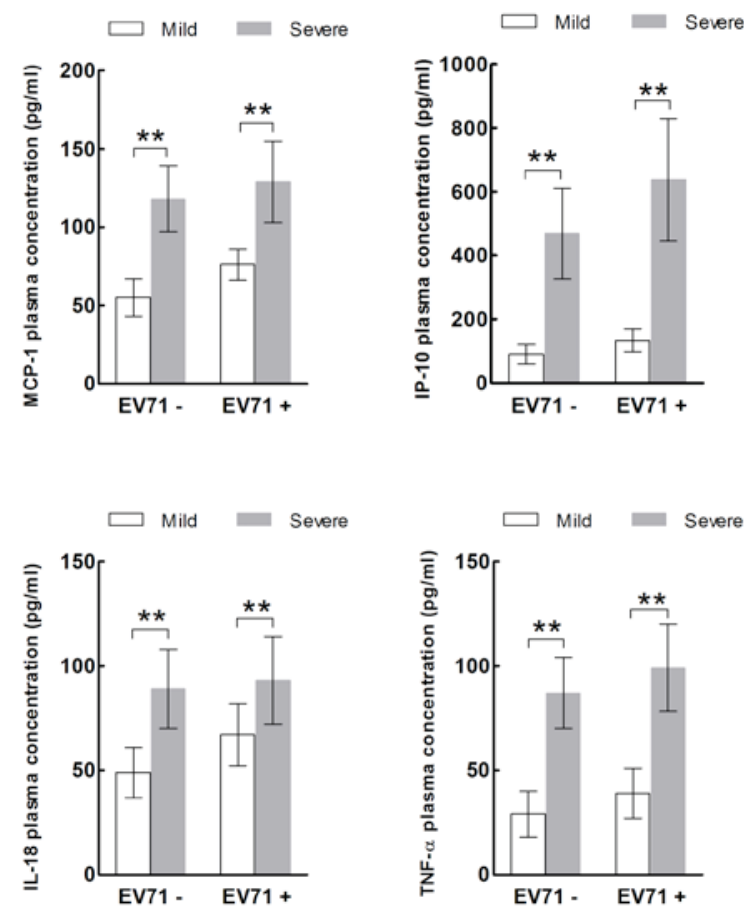

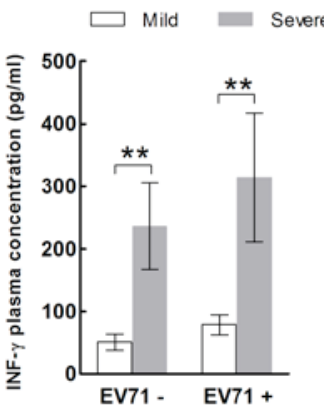

Figure 5. Expression of inflammatory (A) chemokines and (B) cytokines in the EV71-positive and EV71-negative groups according to severity of hand, foot and mouth disease. ${ }^{* *} \mathrm{P}<0.01$. EV71, enterovirus 71 ; IL, interleukin; RANTES, regulated on activation, normal T cell expressed and secreted; MCP-1, monocyte chemoattractant protein-1; IP-10, IFN- $\gamma$-inducible protein-10; TNF- $\alpha$, tumor necrosis factor- $\alpha$; IFN- $\gamma$, interferon- $\gamma$.

\section{References}

1. Huang X, Wei H, Wu S, Du Y, Liu L, Su J, Xu Y, Wang H, Li X, Wang Y, et al: Epidemiological and etiological characteristics of hand, foot and mouth disease in Henan, China, 2008-2013. Sci Rep 5: 8904, 2015.

2. Solomon T, Lewthwaite P, Perera D, Cardosa MJ, McMinn P and Ooi MH: Virology, epidemiology, pathogenesis, and control of enterovirus 71. Lancet Infect Dis 10: 778-790, 2010.

3. Li J, Huo X, Dai Y, Yang Z, Lei Y, Jiang Y, Li G, Zhan J and Zhan F: Evidences for intertypic and intratypic recombinant events in EV71 of hand, foot and mouth disease during an epidemic in Hubei Province, China, 2011. Virus Res 169 195-202, 2012.

4. Yang L, Hu J and Zhou Z: Central nervous system damage associated with hand-foot-mouth disease induced by enterovirus 71 J Appl Clin Pediatr 23: 1782-1785, 2008.

5. Ooi MH, Wong SC, Lewthwaite P, Cardosa MJ and Solomon T: Clinical features, diagnosis, and management of enterovirus 71. Lancet Neurol 9: 1097-1105, 2010.

6. The Ministry of Health of the People's Republic of China: Guideline for the diagnosis and treatment of hand foot and mouth disease, Beijing, China, 2010. Int J Respir 30: 1473-1475, 2010.

7. Clinical Experts Group of the Ministry of Health for Hand, Foot and Mouth Disease: Experts consensus on rescue and treatment of severe cases with enterovirus 71 (EV71) infection. Zhonghua Er Ke Za Zhi 49: 675-678, 2011 (In Chinese).
8. Lin JY and Shih SR: Cell and tissue tropism of enterovirus 71 and other enteroviruses infections. J Biomed Sci 21: 18, 2014

9. Chang LY, Hsiung CA, Lu CY, Lin TY, Huang FY, Lai YH, Chiang YP, Chiang BL, Lee CY and Huang LM: Status of cellular rather than humoral immunity is correlated with clinical outcome of enterovirus 71. Pediatric Res 60: 466-471, 2006.

10. Lin TY, Chang LY, Huang YC, Hsu KH, Chiu CH and Yang KD: Different proinflammatory reactions in fatal and non-fatal enterovirus 71 infections: Implications for early recognition and therapy. Acta Paediatr 91: 632-635, 2002.

11. Wang SM, Lei HY, Yu CK, Wang JR, Su IJ and Liu CC: Acute chemokine response in the blood and cerebrospinal fluid of children with enterovirus 71-associated brainstem encephalitis. J Infect Dis 198: 1002-1006, 2008.

12. Livak KJ and Schmittgen TD: Analysis of relative gene expression data using real-time quantitative PCR and the 2(-Delta Delta $\mathrm{C}(\mathrm{T}))$ method. Methods 25: 402-408, 2001.

13. Wang SM, Lei HY, Huang KJ, Wu JM, Wang JR, Yu CK, Su IJ and Liu CC: Pathogenesis of enterovirus 71 brainstem encephalitis in pediatric patients: Roles of cytokines and cellular immune activation in patients with pulmonary edema. J Infect Dis 188: 564-570, 2003

14. Napolitano LM: Immune stimulation in sepsis: To be or Not to be? Chest 127: 1882-1885, 2005.

15. van den Berghe G, Wouters P, Weekers F, Verwaest C, Bruyninckx F, Schetz M, Vlasselaers D, Ferdinande P, Lauwers P and Bouillon R: Intensive insulin therapy in the critically ill patients. N Engl J Med 345: 1359-1367, 2001 
16. Lekkou A, Karakantza M, Mouzaki A, Kalfarentzos F and Gogos CA: Cytokine production and monocyte HLA-DR expression as predictors of outcome for patients with community-acquired severe infections. Clin Diagn Lab Immunol 11: 161-167, 2004.

17. Wan S, Xia C and Morel L: IL-6 produced by dendritic cell from lupus-prone mice inhibits CD4+CD25+ T cell regulatory functions. J Immunol 178: 271-279, 2007.

18. Woods PS, Tazi MF, Chesarino NM, Amer AO and Davis IC: TGF- $\beta$-induced IL- 6 prevents development of acute lung injury in influenza A virus-infected F508del CFTR-heterozygous mice. Am J Physiol Lung Cell Mol Physiol 308: L1136-L1144, 2015.

19. Wang SM, Lei HY, Huang MC, Su LY, Lin HC, Yu CK, Wang JL and Liu CC: Modulation of cytokine production by intravenous immunoglobnlin in patients with enterovirus 71-associated brainstem encephalitis. J Clin Viral 37: 47-52, 2006.

20. Wang SM, Lei HY, Su LY, Wu JM, Yu CK, Wang JR and Liu CC: Cerebrospinal fluid cytokines in enterovirus 71 brain stem encephalitis and echovirus meningitis infections of varying severity. Clin Microbiol Infect 13: 677-682, 2007. 